\title{
Generation of anti-Giardia antibodies by bacteriophage antibody display
}

\author{
Victoria C. Hough ${ }^{\mathrm{a}, *}$, John Greenman ${ }^{\mathrm{b}}$ and \\ Timothy A. Paget ${ }^{\mathrm{a}}$ \\ Departments of ${ }^{\mathrm{a}}$ Biological Sciences and ${ }^{\mathrm{b}}$ Surgery, \\ University of Hull, Hull HU6 7RX, UK
}

\section{Introduction}

Giardia intestinalis is a protozoan parasite which infects the gastrointestinal tract of man and animals. It is endemic throughout the world and is transmitted via faecal contamination of drinking water and food. $G i$ ardia intestinalis has a relatively simple life cycle alternating between the trophozoite (proliferative form) and the cyst (resting form). The nature of symptoms associated with Giardia infections varies between individuals with many cases being asymptomatic, however in symptomatic cases diarrhoea, weight loss, and abdominal cramps are commonly reported [1].

Treatment of Giardia typically involves a number of drugs belonging to the $5^{\prime}$ nitroimidazoles. Metronidazole (1- $\beta$-hydroxyethyl-2-methyl-5-nitroimidazole $)$ is the most commonly used as it is generally well tolerated [2]. In most cases Giardia responds well to treatment, however in 1-4\% of cases drug resistance is responsible for treatment failure.

Several mechanisms of drug resistance have been investigated in the parasitic protozoa, including drug inactivation via redox cycling and improved drug efflux. Here we describe the use of phage antibody technology to probe the surface of Giardia to identify changes between metronidazole resistant and sensitive trophozoites, and to identify molecules which may confer resistance. We have also used this technology to generate antibodies against Giardia cysts.

*Correspondence to: Miss Victoria Hough, University of Hull, Department of Biological Sciences, Hardy Building, Cottingham Road, Hull HU6 7RX, UK. Fax: +44 1482 466033; E-mail: V.C.Hough@ medschool.hull.ac.uk.

\section{Methods}

\subsection{Use of the bacteriophage antibody display library}

A human synthetic scFv Library \#1 (Nissim library) [3] was obtained from Dr. G. Winter (MRC Centre for protein engineering, Cambridge). This library has more than $10^{8}$ specificities consisting of a single $\mathrm{V} \lambda 3$ light chain gene segment paired with an array of in vitro rearranged $\mathrm{V}_{H}$ gene segments [3].

\subsection{Selection on Giardia trophozoites}

To isolate antibody fragments that were specific to metronidazole resistant Giardia trophozoites, panning was undertaken as shown in Fig. 1. The trophozoites used in the positive rounds of panning were JKH-1, a metronidazole resistant strain. A laboratory strain that had not been cultured in the presence of metronidazole was used for the negative round of panning. Prior to panning the trophozoites were fixed in methanol to prevent motility, and blocked in milk powder/BSA buffer to prevent non-specific binding of the bacteriophage.

\subsection{Selection on Giardia cysts}

BV-M and MR-4 Giardia cysts were used for positive selection and Acanthamoeba cysts were used for the subtractive panning round. Four rounds of selection were undertaken in total, after which phage were selected at random for further study.

\subsection{Whole cell ELISA}

Supernatant containing single phage clones chosen at random after the final positive selection was added to methanol fixed target trophozoites in conical bottomed 96 well plates. After washing, binding was assayed by the addition of HRP-conjugated anti-M13 (Pharmacia) and ABTS was used as substrate. Absorbance was measured at 405-690 nm after transfer to flat bottomed plates. The negative controls were an anti-NIP bacteriophage clone and no bacteriophage. 


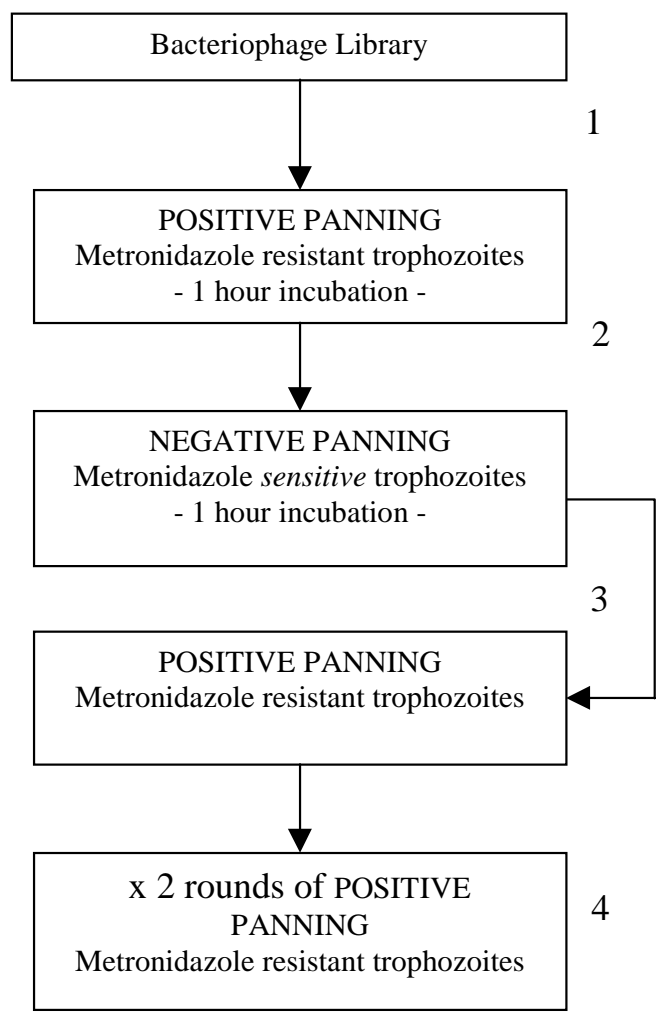

Fig. 1. Panning protocol for selection on Giardia Trophozoites. Initially $1 \times 10^{13}$ bacteriophage were added to metronidazole resistant trophozoites, $2 \times 10^{9}$ bacteriophage were removed and amplified in E.coli TG1 (1). $6.2 \times 10^{12}$ bacteriophage were then added to metronidazole sensitive trophozoites for a subtractive selection step (2), the unbound bacteriophage were immediately incubated with metronidazole resistant trophozoites (3). After elution and amplification in E.coli TG1 the bacteriophage were then used in 2 further rounds of positive selection (4).

\subsection{Flow cytometry}

Clones that bound strongly by ELISA were selected for further study using flow cytometry. Trophozoites were incubated with the test phage clone and detected using an anti-M13 monoclonal antibody (Amersham Pharmacia Biotech) followed by a FITC-conjugated anti-mouse polyclonal antibody. Cells were analysed using a FACSCalibur flow cytometer with CellQuest software.

\section{Results}

\subsection{Selection on metronidazole resistant Giardia trophozoites}

300 clones were analysed by ELISA. Sixteen clones that showed variable binding to JKH-1 were chosen.
Table 1

Binding of selected bacteriophage to Giardia trophozoites

\begin{tabular}{lcccc}
\hline Clone & BV-M & JKH-1 & MR-4 & NESS \\
\hline A2 & $15.6 \%$ & - & - & 0 \\
E1 & $14.0 \%$ & - & - & 0 \\
F2 & $13.9 \%$ & - & - & $24.1 \%$ \\
F7 & $33.1 \%$ & $15.1 \%$ & - & $23.1 \%$ \\
G2 & $17.5 \%$ & - & - & $30.9 \%$ \\
G3 & $14.0 \%$ & - & - & $34.3 \%$ \\
G12 & $21.9 \%$ & $13.5 \%$ & - & $33.3 \%$ \\
H8 & $15.9 \%$ & $13.1 \%$ & - & $32.2 \%$ \\
AD5 & $16.0 \%$ & $16.3 \%$ & - & $29.0 \%$ \\
AE2 & $37.5 \%$ & $13.5 \%$ & - & $24.2 \%$ \\
AE3 & $31.5 \%$ & - & - & $30.5 \%$ \\
BE3 & $16.7 \%$ & - & - & $31.4 \%$ \\
BE5 & $19.8 \%$ & $18.4 \%$ & - & $34.2 \%$ \\
BF4 & $15.0 \%$ & $18.5 \%$ & - & $29.2 \%$ \\
BF12 & $22.5 \%$ & $14.0 \%$ & - & $33.6 \%$ \\
BH11 & $25.5 \%$ & - & - & $38.2 \%$ \\
\hline
\end{tabular}

Table 2

Binding of selected bacteriophage clones to Giardia cysts

\begin{tabular}{lcccc}
\hline & \multicolumn{4}{c}{ Giardia cyst strains } \\
\cline { 2 - 5 } Clone & BV-M & JKH-1 & MR-4 & NESS \\
\hline A10 & - & $18.0 \%$ & $36.9 \%$ & - \\
B11 & - & - & - & - \\
D3 & - & $24.3 \%$ & $14.5 \%$ & $18.3 \%$ \\
D4 & - & - & $26.3 \%$ & $35.3 \%$ \\
F1 & - & - & $17.8 \%$ & $11.4 \%$ \\
F5 & - & $18.3 \%$ & $19.2 \%$ & $10.6 \%$ \\
H2 & - & - & - & - \\
H3 & - & - & $29.0 \%$ & - \\
\hline
\end{tabular}

CDR3 insert size was determined by PCR using previously described primers [3]. Flow cytometry was performed on four strains of Giardia, percentage binding to each strain can be seen in Table 1 .

\subsection{Selection on Giardia cysts}

200 clones were analysed by ELISA. 8 clones were selected for study by flow cytometry on the basis of strong binding to MR-4 or JKH1 cysts. The degree of binding to each of 4 laboratory derived cysts can be seen in Table 2. Fluorescent microscopy was used to confirm the flow cytometry results.

\section{Conclusions}

In this study we have shown that it is possible to generate antibody fragments which bind to Giardia trophozoites and cysts. The clones show variable levels of binding and cross reactivity to different strains. Clone H3 appears specific for cysts from the MR-4 strain 
whereas all other clones showed cross-reactivity with two or three trophozoite or cyst strains, as appropriate.

In the future we aim to select clones which are specific to metronidazole resistant trophozoites after additional rounds of cell selection. The existing clones will be used to characterise surface molecules and to determine any role(s) in resistance. We also plan to investigate possible differences in the surface structure of animal and human trophozoites.

\section{References}

[1] P.F.L. Boreham, Giardiasis and its control, The Pharmaceutical Journal 31 (1991), 271-274.

[2] C.D. Freeman, N.E. Klutman and K.C. Lamp, Metronidazole a therapeutic review and update, Drugs 54(5) (1997), 679-708.

[3] A. Nissim, H.R. Hoogenboom and I.M. Tomlinson et al., Antibody fragments from a "single pot" phage display library as immunochemical reagents, EMBO, J. 13 (1994), 628-629. 


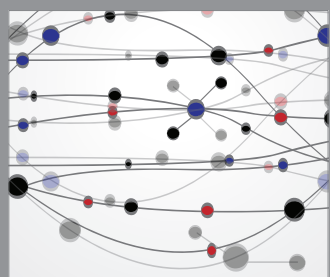

The Scientific World Journal
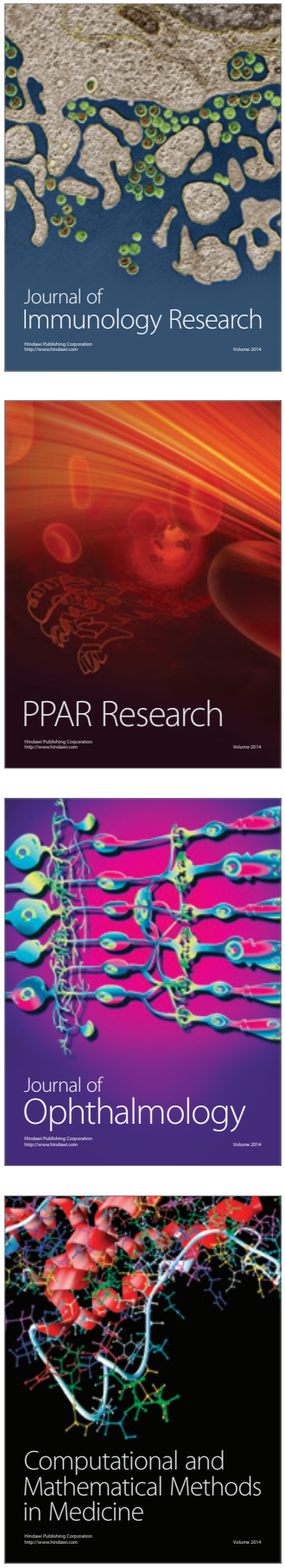

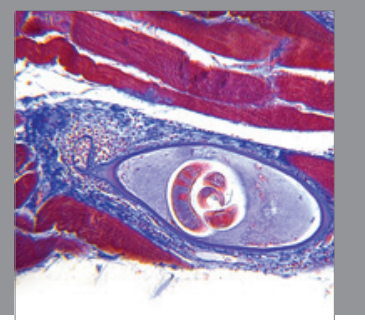

Gastroenterology

Research and Practice
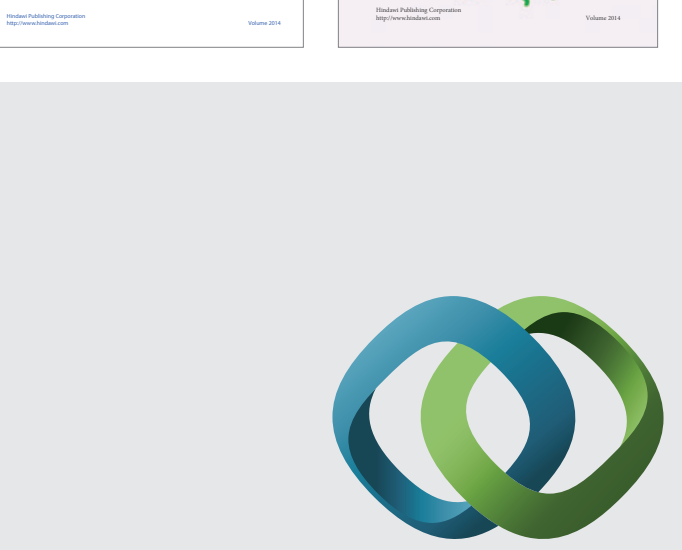

\section{Hindawi}

Submit your manuscripts at

http://www.hindawi.com
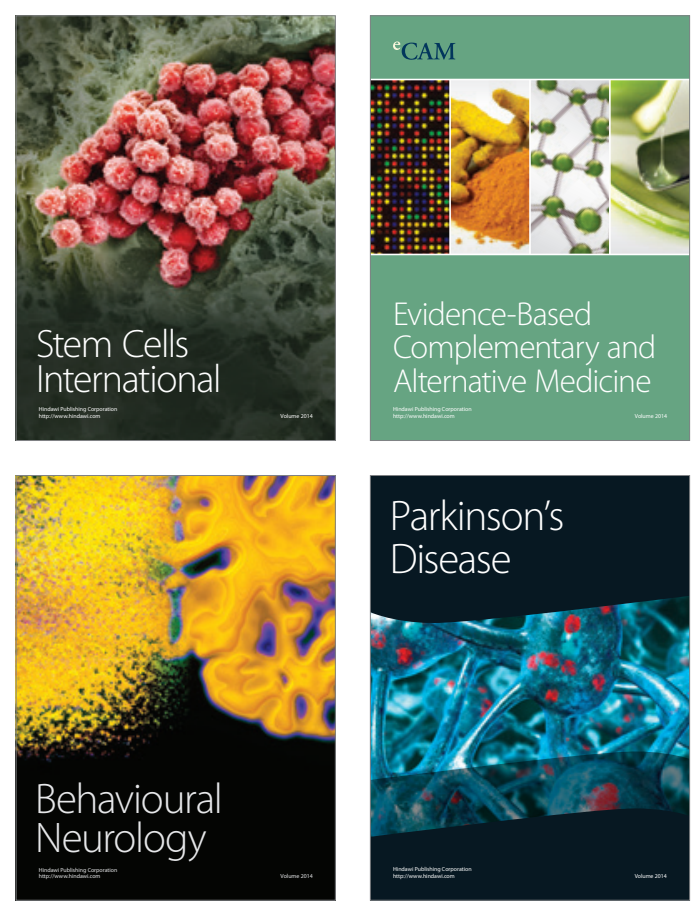

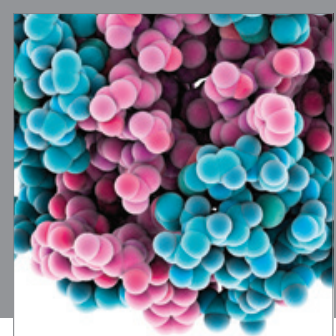

Journal of
Diabetes Research

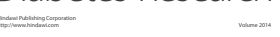

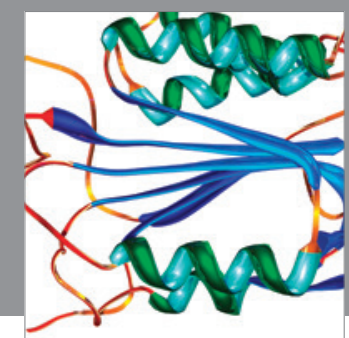

Disease Markers
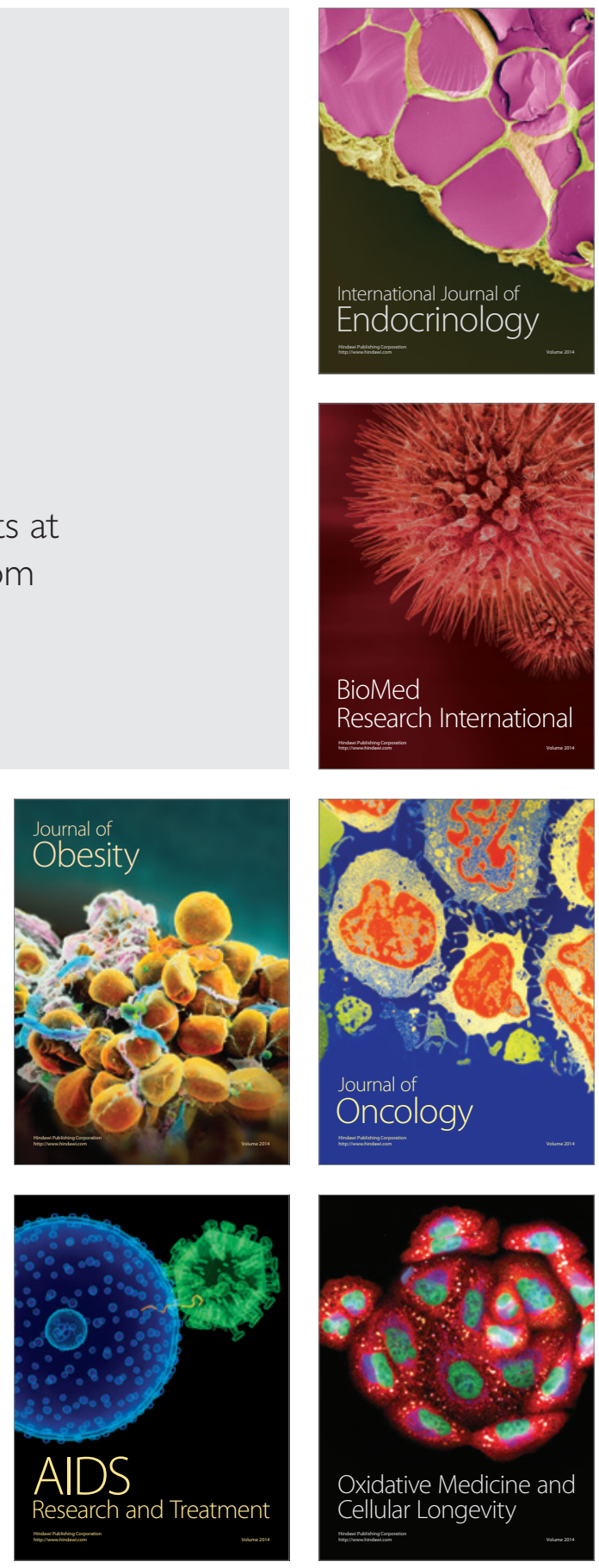